\title{
La educación que deja huella: descripción del trabajo de campo en la población de Magueyitos, Quimixtlán
}

\author{
Angélica Espina Pérez \\ Licenciada en Administración de Empresas. Antropóloga social. \\ Profesora de Historia y emprendedora mexicana. \\ e-mail: a_n_gy246@hotmail.com
}

\section{Resumen}

El tema de la educación es un gran detonante para diversas investigaciones realizadas a lo largo de la historia y ha estado en auge durante los últimos años. El interés de este tema se identifica en las distintas disciplinas científicas como: la sociología, la historia y la filosofía por mencionar algunas; por lo que, la antropología no podía quedarse rezagada. El presente texto tiene por objetivo reseñar el trabajo de campo realizado en Magueyitos-Quimixtlán en el Estado de Puebla, México; enfocado en la educación del nivel básico-primaria. Se presenta una descripción densa de la educación en las escuelas rurales; el acoplamiento de los docentes, alumnos y tutores ante las dificultades económicas, materiales, tecnológicas y sociales al que se enfrentan continuamente. Además, se analiza las diferencias y similitudes entre las escuelas urbanas y rurales; al conocer el rol de los profesores y el acceso limitado a los servicios básicos que demuestran la ausencia del Estado. Sin embargo, la esperanza de los padres y tutores, sobre la educación como el trampolín para mejorar la calidad de vida de los educandos se observa de manera teórica y práctica.

\section{Palabras clave}

Antropología, educación, trabajo de campo, relación docente - alumnos. 


\title{
Education that leaves a mark: description of field work in the town of Magueyitos, Quimixtlán
}

\author{
Angélica Espina Pérez \\ Mexican Social Anthropologist. Degree in Business Management. \\ History teacher and entrepreneur. \\ e-mail: a_n_gy246@hotmail.com
}

\begin{abstract}
The issue of education is a great trigger for various investigations carried out throughout the history of the human being, this has been on the rise in recent years. The interest in this topic is identified in the different scientific disciplines such as: sociology, history and philosophy to name a few; therefore, anthropology could not lag behind. The present text aims to review the field work carried out in MagueyitosQuimixtlán in the State of Puebla, Mexico focused on education at the basic-primary level. A dense description of education in rural schools is presented; the coupling of teachers, students and tutors in the face of economic, material, technological and social difficulties that they continually face. In addition, the differences and similarities between urban and rural schools are analyzed; knowing the role of teachers and the limited access to basic services that demonstrate the absence of the State. However, the hope of parents and guardians about education as the springboard to improve the quality of life of students is observed in a theoretical and practical way.
\end{abstract}

\section{Keywords}

Anthropology, education, field work, teacher-student relationship. 


\section{Introducción}

El siguiente texto pertenece al trabajo de campo realizado en Magueyitos-Quimixtlán ubicado en el Estado de Puebla, al cursar la licenciatura en Antropología Social en la Benemérita Universidad Autónoma de Puebla (BUAP), en el año 2011, cuando ingresé al Seminario de Tesis I para analizar el tema de "La educación en México". En ese momento, me desempeñaba como docente titular en una institución primaria particular del Estado de Puebla, donde surgió la oportunidad de participar en el curso piloto desarrollado por la Secretaria de Educación Pública denominado "Programa de Atención Intensiva (PAI)". Dicho curso se dio a conocer por medio de carteles de avisos de las diferentes instituciones públicas y privadas que participaban. Consistía en acudir a impartir clases a niños de 5 y 6 años, que cursan el primero y segundo grado del nivel primaria, de las diferentes comunidades del Estado de Puebla durante el periodo vacacional de verano. El objetivo fue regularizar a los alumnos que presentaban problemas de rezago escolar mediante estrategias lúdicas, que les permitiera ingresar al nuevo ciclo escolar con un conocimiento significativo y estar a la par de sus compañeros, en base al contenido establecido por los programas educativos de México.

La motivación de participar en el programa (PAI) fue para adquirir conocimientos nuevos y experiencia profesional al permitir conjugar de manera diferente y acertada mi desarrollo en lo laboral como docente titular y en lo intelectual como antropóloga. Esto, a través de la enseñanza de nuevos conocimientos enfocados a una realidad desigual en el área educativa, social y material; lo cual se vería plasmado en el Seminario de Tesis I. El análisis de las diferentes realidades que se pueden observar en una comunidad desde la perspectiva antropológica y pedagógica permite comprender de mejor manera las realidades educativas que se perciben.

Me asignaron la comunidad de Magueyitos, localizada en el municipio de Quimixtlán (Estado de Puebla) en dirección al este con 3.2 kilómetros que colinda con la Sierra Madre oriental. Magueyitos, cuenta con un aproximado de 341 habitantes y 56 viviendas de las cuales el $60 \%$ de la población es indígena. La lengua más hablada es el náhuatl, los habitantes de 6 a 25 años son generalmente bilingües hablan español y náhuatl; pero, solo escriben en español. La mayoría de las viviendas carece de servicios básicos como electricidad, agua potable, desagüe; ya que las casas están construidas a distancias lejanas entre ellas (350 a $500 \mathrm{~m}$ aproximadamente) lo que dificulta la realización de obras públicas. Sin embargo, esto no les desalienta en el desarrollo de sus actividades. La principal actividad económica es la agricultura a pequeña escala. Cultivan café, maíz, frijol y calabaza; así como plantas frutales de manzanas, duraznos y capulíes.

Conforme avanzan los años, las transformaciones económicas, educativas y socioculturales planteadas por el Estado en sus políticas y reformas constitucionales tienen un resultado limitado y contradictorio. Esto último se evidencia en los 
programas sociales, cuyo objetivo es el de impulsar el avance de las comunidades rurales y sus pobladores. Por otro lado, promueven el crecimiento de los bienes privados individualmente con la tendencia de aumentar la desigualdad social, la extrema pobreza de los habitantes y la falta de servicios básicos, educación y salud digna haciendo visible la estrecha vinculación entre marginación y ruralidad.

\section{El camino hacia Magueyitos}

Hasta ese momento, no tenía conocimiento de la ubicación ni la forma de llegar a Magueyitos. Al investigar, en diversas fuentes y recomendaciones de amigos, logré conseguir y coordinar la ruta hacia la comunidad días antes de mi partida. Un día antes del viaje, organicé mi equipaje y, a las 6 de la mañana, salí hacia la comunidad desde la CAPU (Central de Autobuses de Puebla). El recorrido consistía en llegar primero a San Francisco Quimixtlán en un tiempo aproximado de dos horas y media en camión. En el kilómetro 150 está el municipio de Amozoc en el Estado de Puebla donde se observa un paisaje con contraste verde - seco y comunidades rurales con un aspecto comunitario distinto. Esto me llevó a realizar una reflexión antropológica enfocada a la desigualdad social existente entre las zonas urbanas y rurales que visibilizaban la falta de acceso a servicios básicos y por precarias obras públicas.

Al llegar a la comunidad San Francisco de Quimixtlán, dentro del terminal de autobuses, tuve que abordar otro colectivo para llegar a la comunidad de Magueyitos. El transporte era una camioneta de batea adaptada con bancas de madera en la parte trasera y tubos metálicos alrededor, que servía como soporte de seguridad para los pasajeros; todo esto cubierto con lona de plástico. El camino no estaba asfaltado, lo que dificultaba el acceso a la comunidad, sobre todo en las temporadas de lluvia, ya que la presencia de neblina que no permite ver a larga distancia. La tierra era como chicle (arcilla; color café claro, mojado presenta plasticidad y seca dureza). De un lado, se tenía el cerro y del otro extremo se encontraba un barranco. Por ello, el transporte tenía que ir con mucho cuidado a una velocidad mínima.

En el terminal, solo había dos turnos al día: el transporte del primer turno salía a las 12 p.m. y, el segundo a las 4 de la tarde. Abordé el segundo turno cuyo conductor asignado era Miguel, a quien los pasajeros llamaban "don Miguel". Antes de partir, don Miguel me explicó que no llegaba hasta la comunidad de Magueyitos, ya que, la carretera resulta compleja por la dimensión y el periodo de tiempo sería un aproximado de 2 horas y media. "¿Ve usted esos dos cerros?”, dijo don Miguel mientras señala con su dedo índice los cerros que veían frente a nosotros, "pues Magueyitos está en el otro cerro (el más lejano) vamos a llegar a un crucero donde termina mi ruta; allí, usted esperará a que alguien pase y le pedirá que la lleve a Magueyitos. Es como una hora más, pues solo se llega a caballo o remolque".

Esperé cerca de 40 minutos para partir mientras llegaban más pasajeros, los cuales se saludaban mutuamente y conversaban amigablemente. Al llegar la hora de 
iniciar el recorrido, las personas que viajaban en la unidad me preguntaron; “DDe dónde eres? ¿A qué vienes?; mi respuesta fue "soy antropóloga y daré clases en Magueyitos a los niños de primaria". Ellos me dijeron que los profesores asignados a esa comunidad asistían pocas veces por distintas razones (comisiones, distancia, entre otras). Los alumnos casi no tenían clases. Los niños se dedicaban a trabajar desde muy temprana edad en el campo. Por otro lado, gran parte de los pobladores mayores de 30 años no sabían leer ni escribir y hablan náhuatl, se dedican a labores como recolectar café, sembrar maíz y los jóvenes emigraban hacia la capital (Ciudad de México) para obtener más recursos económicos. Cuando llegué a mi destino, todos se despidieron de mí. Esperé a que alguien pasara por el crucero el cual estaba totalmente despoblado.

Después de una hora de espera aproximadamente, pasó “don Mateo", acompañado de su burro. Lo saludé y me preguntó: “¿A dónde se dirige?”, “a la escuela primaria de Magueyitos", le contesté y muy gentilmente me dijo: "yo la llevo". Subí el equipaje y empezamos a caminar. Me preguntó si yo era maestra, le dije que sí, que enseñaría a los niños de la primaria. Don Mateo comentó que él es originario de Magueyitos donde antiguamente no existían escuelas; de modo que no sabía leer ni escribir. Sin embargo, le alegraba que en la actualidad los niños tuvieran la oportunidad de estudiar. Mencionó que antes había un maestro llamado Miguel quien desde joven (15 o 16 años) llego a impartir clases y se quedó a vivir en la comunidad por muchos años. Sobre ello, mencionó:

Nosotros lo alimentamos, lo vimos crecer; Pues, cuando llegó estaba todo menudito y cuando se fue ya hasta hijos tenía; pero, como no vivían con él se fue a su tierra. A los niños les enseñó a bailar danza, organizaba la fiesta patronal era un buen muchacho; Pero, desde que se fue jamás lo hemos vuelto a ver. (Don Miguel).

\section{El encuentro}

Los padres de familia me saludaron dándome la bienvenida. Me invitaron a comer en el salón de clases; en cuyo escritorio, yacía una olla de barro con frijoles negros en caldo con epazote, en otra olla de peltre, café de grano. Todo ello servido en un plato y jarro de barro; además, había tortillas hechas a mano envueltas en una servilleta de tela con una imagen de un perro bordado a mano. Los niños se sentaron a mi lado y sus padres o abuelos frente a mí. Mientras comía, me platicaron sobre la profesora encargada de la escuela, "ella se encuentra con nosotros desde hace 10 años, es del municipio de Libres, allá tiene su familia y se va todos los viernes para verlos y regresa el lunes muy temprano." Por otro lado, los niños me preguntaron si tenía hijos y cómo era donde vivía.

Después de comer, recordé el agradable aroma a humo generado por la leña de los fogones. El epazote combinado con el olor del café me hizo recordar mi infancia en casa de mi abuela paterna. Más tarde, me explicaron que los niños me traerían el 
desayuno al salón de clases, cada familia estaría encargada de prepararlo; es decir, se iría rotando entre todas las casas (aproximadamente 25), cada niño me recogería en la escuela para llevarme a sus casas al concluir las clases. Finalmente, me mostraron el salón que sería mi habitación de descanso, la cual tenía un catre, una mesa y silla de madera.

La escuela lleva por nombre "Francisco I. Madero", era la única institución educativa en la comunidad. Consta de cuatro salones de clases, de las cuales dos son usadas como cuarto de hospedaje para la profesora principal, que era la directora al mismo tiempo (solo estaba ella para impartir las clases de todos los grados y dirigir la institución); pero también, había una aula de dirección y una cancha de básquetbol. La mayoría de los habitantes eran personas de la tercera edad, mujeres y niños. Los hombres adultos generalmente salían del pueblo en busca de un trabajo mejor remunerado. Las casas eran de madera con techo de lámina, solo las que estaban cerca de la escuela tenían electricidad y agua potable; la carencia de acceso a estos servicios básicos representa la débil presencia del Estado, en el desarrollo de obras públicas en beneficio de la población. Los caminos que había eran de tierra y para poder llegar a algunas casas se caminaba sobre veredas naturales que rodeaban los sembríos, arroyos y cerros.

Para iniciar activamente con mis actividades, programé una junta de padres y/o apoderados para el día siguiente, donde debía exponer el plan de trabajo a desarrollarse durante mi estadía; además, esto me permitiría conocer sus inquietudes y expectativas, para poder identificar las oportunidades y fortalezas de los alumnos y de la comunidad.

\section{Entre clases y la vida cotidiana}

Al día siguiente, muy puntual a las 8:00 a.m., se encontraban los tutores y alumnos en la cancha deportiva esperando iniciar la reunión programada. En primer lugar, expliqué el plan de trabajo y su objetivo; de reforzar los conocimientos vistos en el ciclo escolar concluido de primero y segundo grado de primaria con el método constructivista $^{1}$, el cual expuse de manera clara y detallada. Asimismo, les facilité pautas a los tutores para apuntar sus dudas o propuestas.

Los alumnos de la lista proporcionada por el asesor del programa eran 8. Una de las solicitudes fue que todos los niños de la comunidad pudiesen asistir a la escuela; serían en total 25 alumnos de todos los grados (de primero a sexto grado). Esta idea me agradó y acepté positivamente. El horario de clases sería de lunes a viernes de 8:00 a.m. a 1:00 p.m., y abría un tiempo de descanso a las once para que los niños disfruten de sus refrigerios y puedan convivir entre ellos. Algunos alumnos

\footnotetext{
1 Método pedagógico, basado en entregar herramientas a los estudiantes que les permitan construir su conocimiento y propios procedimientos para resolver problemas.
} 
caminaban cerca de una hora desde sus casas a la escuela; por lo que, se les permitía el ingreso después de la hora establecida como entrada. Tenían que asistir con su uniforme de la escuela y debían traer los materiales didácticos proporcionados por la SEP y por mi cuenta (libros, lápiz, libretas, cuadernos); esto materiales los repartía todos los días antes de iniciar la clase para no generar un costo extra a sus tutores.

Por otro lado, para los alumnos de sexto grado posiblemente era su último curso educativo, por las dificultades que genera continuar sus estudios secundarios. Por ellos, es necesario mencionar estas dificultades: tenían que caminar cerca de dos horas y media para llegar al colegio. Lo cual representaba un peligro para ellos: debían salir en la madrugada, aun cuando no había luz del sol, y por la tarde debían regresar temprano. Esto significaba que los estudiantes estén expuestos a cualquier accidente, de algún animal. Por otra parte, es innegable que la economía de sus tutores no es suficiente para cubrir la compra de uniformes, materiales didácticos y gastos extras que se presentarán; motivo por el cual la mayoría de los alumnos se quedan en el nivel primario.

Los padres de familia y tutores exponían de manera oral y expresiva su interés por la educación de los niños de la comunidad (hijos, nietos, sobrinos, vecinos). La mamá del alumno Kevin dijo: "Es la única manera de que nuestros hijos tengan una vida mejor y salgan adelante o de este pueblo". Su comentario fue apoyado por los demás padres que movían la cabeza en señal de acuerdo.

Con el paso del tiempo, se tomó un examen de diagnóstico en el cual se identificó que los alumnos de sexto grado no sabían leer de manera correcta (deletreaban o no identificaban las letras), ni lograban resolver problemas matemáticos. Los niños de primer año no identificaban correctamente las vocales, números ni colores. Esto se debía a muchos factores como: la alimentación, ya que la gran mayoría de los niños asistían sin desayunar o sus almuerzos eran una taza de café, así como la falta de tiempo dedicado al estudio; pues los niños desde muy pequeños apoyaban a sus padres en labores agrícolas. Los tutores en su mayoría eran adultos mayores que requerían el apoyo de los niños, estos se levantaban temprano para realizar labores de la casa (recogían leña, limpiaban los animales, recogían frutos en el campo, etc.)

\section{Los casos del alumno $A$ y alumno $B^{2}$}

Un caso significativo que representa las dificultades educativas de la comunidad es el del alumno A, había concluido el tercer grado de primaria con una calificación reprobatoria. Indagando más sobre la vida del niño, descubrí que vivía con su abuelita materna, quien era invidente, su mamá trabajaba en la capital (Ciudad de México), y solo los visitaba dos veces al año.El alumno A, antes de asistir a la escuela, que

Esta sección contiene el caso de dos niños menores de edad, por temas de confidencialidad y por respeto a los informantes, es necesario la protección de sus identidades. Por lo que, me referiré a ellos como alumno A y alumno B. 
se encontraba a una hora y media de camino, tenía que dejar aseado el lugar donde se encontraban los animales (pollos y borregos), debía preparar el desayuno para su abuela, recolectar leña si fuera necesario y acarrear el agua del río (que pasaba aproximadamente a 10 minutos de su casa). Después de dichas labores, asistía a la escuela. Por la tarde, continuaba con sus deberes y el cuidado de su abuelita. Su horario de dormir era cerca de las 7 de la noche, pues no contaban con luz eléctrica. Tampoco tenían agua potable ni desagüe y siempre cocinaba a leña. Al platicar con él me decía que extrañaba mucho a su mamá y que no realizaba las tareas porque no le daba tiempo y en la noche se sentía muy cansado.

Otro ejemplo que evidencia la falta de recursos tecnológicos es el caso del alumno B, él tenía un excelente promedio; por ello, la Secretaria de Educación Pública le regaló una tablet. Pero, debido al difícil acceso a la electricidad y el internet, el alumno B no podía aprovechar este recurso tecnológico. Asimismo, sus padres no sabían utilizar el dispositivo y, por consecuencia, no podían enseñarle a su hijo el manejo de la tablet. Lamentablemente, esta se encontraba en su caja totalmente nueva, sin uso y sin poder ser de ayuda en la educación del alumno B. Estos casos no eran únicos, pues varios alumnos experimentaban las mismas condiciones derivadas de la falta de recursos materiales y económicos que tiene la comunidad.

\section{El rol de los docentes}

Definitivamente el rol de un profesor en las comunidades rurales es importante. Son parte de la comunidad no solo como docentes, sino también como instructores de vida que generan un pensamiento crítico. Además, forman parte de las actividades cotidianas de los habitantes, especialmente de los alumnos, ya que tienen que conocer a profundidad sus sueños, su vida familiar y su entorno social para poder comprender sus necesidades e impartir un conocimiento que logre fomentar un pensamiento crítico y reflexivo. Para conseguir todo lo mencionado el profesor tiene que estar en un constante aprendizaje, evolucionando sus metodologías y técnicas de enseñanza, como expresa Sánchez:

Los principales objetivos para el éxito del proceso de modernización de la educación se centran en la calidad de la enseñanza, es decir, en la acción y competencias del profesorado, las que se conciben como dirigidas hacia la obtención de objetivos específicos de aprendizaje, enseñanza y desarrollo. (2001).

El respeto y admiración por un profesor está a flor de piel como los tutores y el resto de la comunidad lo menciona, los docentes "son las personas que toman la mano de sus hijos, los guían e impulsan para ser mejores y conocer el mundo de otra manera", en estas líneas Villarroel y Thomas, mencionan que el papel del profesor rural, por tanto, no se limita sólo a la relación profesor-alumno, pues su labor se concibe como un servicio a la comunidad (en Vera et al., 2012). 
Sin embargo, a este en su formación, no se le menciona las múltiples tareas y funciones que debe desempeñar en su lugar de trabajo; lo cual, genera afectaciones en el tiempo, así como en la motivación de enseñar, la misma que se refleja en sus propios alumnos, como expresan Carlos Marcelo y Denise Vaillant (2009): "Plantean que los docentes actuales expresan una identidad profesional que transita entre un sentido misionero y una perspectiva de facilitador del aprendizaje, en ocasiones, como profesional reflexivo". La percepción sobre el trabajo realizado por los docentes es valorada desigualmente. En las escuelas privadas no se proporciona respeto al trabajo que realizan los docentes; quienes, se ven como los responsables totales de la educación y el cuidado de los niños; sin el apoyo de los tutores al realizar tareas, trabajos o una retroalimentación en la conducta del estudiante. En cuanto a las escuelas rurales les hace falta material didáctico, apoyo administrativo y presión para cumplir con los programas educativos y aunque los padres de familia los apoyen, esto no es suficiente por la falta de conocimiento que tienen. En suma, se vive en una misma temporalidad pero se percibe como si fuera una época diferente.

\section{Escuela rural - escuela urbana}

Existe una gran diferencia entre las escuelas rurales y urbanas, así como entre las escuelas públicas y privadas. Para Kauffaman (1967), el propósito de las escuelas públicas o comunales es el de enseñar a todo el mudo a leer, a escribir, hacer cuentas y valores morales lo que va a permitir que los alumnos puedan aplicar sus conocimientos (básicos) a su vida cotidiana, logrando facilitarles su relación con la sociedad, principalmente en el campo laboral y transmitir las diferentes perspectivas culturales y sociales que existen en el mundo. La diferencia entre las escuelas privadas y públicas ubicadas en zonas urbanas y rurales cerca del Estado de Puebla es muy notoria; pues las escuelas urbanas, en su gran mayoría, tienen acceso a la tecnología suficiente y el personal necesario: ya sean estos docentes, personal administrativo, de aseo u otros; cuentan con una infraestructura lo cual motiva a los alumnos y docentes.

Por otro lado, se encuentra la realidad de las escuelas rurales, con una infraestructura en condiciones deplorables e inestables que ponen en riesgo la vida de los alumnos y docentes; pero, en algunas comunidades no se cuenta ni siquiera con aulas, las clases se desarrollan al aire libre. En cuanto al acceso y manejo de los medios tecnológicos, las escuelas rurales no cuentan con acceso a internet ni computadoras, y si cuentan, no hay personal capacitado para su manejo. Cabe mencionar que también existen falta de recursos humanos. Es decir, solo hay un docente que desempeña el rol de profesor de primero a sexto grado, además de ser director y personal administrativo.

Para que los niños tengan una educación de calidad e igualitaria es necesario estudiar de manera detallada, realista e individual, considerando el entorno social, cultural. Una de las características de una buena educación es su enfoque intercultural, las 
realidades socioculturales son distintas, el docente debe entender que cada estudiante es particularmente diferente a los demás. Aún existen brechas de desigualdad entre las escuelas rurales y urbanas, lo que significa que debemos seguir trabajando para reducirlas. Como menciona Gaviria, "la deserción escolar se entiende por el abandono del sistema educativo por parte de los estudiantes, provocado por una combinación de factores que se generan tanto en la escuela como en contextos de tipo social, familiar e individual" (en Venegas et al., 2017, p. 236).

\section{Reflexiones en torno al trabajo de campo}

Mi estancia en Magueyitos fue de un mes y medio, tiempo en el cual reflexioné a fondo acerca de las desigualdades y dificultades sociales y educativas que existen en el país de México. Para Schmelkes: "La desigualdad es el principal problema de la educación en México porque impide distribuir calidad. Es necesario que todos tengan los aprendizajes que requieren para vivir una vida digna y que se cumpla el derecho a una educación de calidad" (2015). Esto no quiere decir que la desigualdad educativa sea exclusiva de este país, sino es uno de los mayores problemas en América Latina y el mundo. "Uno de cada dos niños no acaba la escuela primaria, la desigualdad de ingresos económicos aumenta y las tasas de desempleo se incrementa, porque hay un montón de gente que está excluida de la oportunidad de ser educado" (Raimers, 2000). A pesar de los avances tecnológicas y la evolución constante que percibimos, el analfabetismo en México no es rezagado, sino, por el contrario, va en aumento. Esto se evidencia en la falta total de conocimientos, al no asistir a una institución educativa, así como en el conocimiento carente de significado para la vida del alumno. Como consecuencia de esto, no se pueden desarrollar positivamente, además de experimentar la cultura de la obligación. Aunque estos son temas de sobrada literatura basada en investigación documental y de campo por diferentes áreas y disciplinas, residir físicamente rebasa por mucho la percepción que tenía anteriormente sobre la sociedad.

Los habitantes de la comunidad de Magueyitos me brindaron conocimiento significativo y reflexivo acerca de los factores internos y externos que afectan el derecho al acceso a una educación de calidad que les permita forjar un camino para su futuro. "La erradicación de la desigualdad social, educativa y de la pobreza ha sido un objetivo importante en las estrategias de desarrollo en la mayoría de las sociedades modernas" (Tilak, 2002), los cuales cuentan con una historia que se sigue reproduciendo en pleno siglo XXI y continúan afectando a la niñez. Para Galván, el derecho a la educación ha sido un anhelo que en México se encuentra documento desde 1821, cuando nuestro país inició su vida independiente de la Corona Española (2016). El acceso a educación igualitaria es fundamental para el desarrollo no solo de un país sino del mundo; pero siguen existiendo brechas de desigualdad tecnológica, carencia de recursos materiales y económicos; así como la falta de vocación, responsabilidad social y personal por parte de los docentes con sus alumnos y la escasez de los mismos. 
La falta de conocimiento sobre las lenguas originarias (hablar, entender y escribir), el uso y costumbres y sus derechos de las comunidades rurales en la formación profesional de los docentes conlleva a generar indiferencia; por ejemplo, cuando el dictado de clases es en español en su totalidad, solicitando materiales didácticos que son difíciles de conseguir. Por otro lado, los docentes desarrollan sus clases con metodologías didácticas e innovadoras; pero estas no se adecuan al contexto sociocultural de los estudiantes. Para ello, es necesario que el docente conozca y respete las lenguas originarias de las comunidades, ya que el programa educativo mexicano no está adaptado a todos los contextos sociales. Navarro menciona:

Una pregunta legítima que podemos hacernos entonces, es: ¿Hasta qué punto la educación ofrece tal igualdad de oportunidades, es decir, facilita que todos tengan la oportunidad de alcanzar sus objetivos educativos por muy ambiciosos que éstos sean, siempre y cuando sus méritos intelectuales así se lo permitan?. Una condición para que tal igualdad de oportunidades ocurra es que todos puedan acceder al máximo a la educación existente, siendo su única limitación su propia capacidad intelectual (2002, p. 99).

Para los tutores, es frustrante no poder apoyar a sus hijos o nietos, pues no cuentan con los recursos económicos suficientes, viven en zonas marginales donde la mayoría son analfabetos. Sin embargo, esto no los detiene y consideran que "la educación es el escalón para que sus hijos puedan volar y crecer", "si nosotros no lo logramos, no fue por la falta de ganas, sino porque en su momento no tuvimos la oportunidad y las opciones eran totalmente escasas". Como menciona el pedagogo Paulo Freire en su libro "Pedagogía del oprimido":

La educación que se impone a quienes verdaderamente se comprometen con la liberación, no puede basarse en una compresión de los hombres como seres "vacíos" a quienes el mundo "llena" con contenidos; no puede basarse en una conciencia especializada, mecánicamente dividida, sino en los hombres como cuerpos conscientes y en la conciencia intencionada al mundo (Freire, 1970, p.70).

Es importante no solo transmitir conocimiento a los educandos, se debe fomentar un pensamiento reflexivo y crítico que puedan aplicar al entorno social en el que se desarrollan, dejando en claro que la educación no es una obligación, es una oportunidad que permitirá que se abran muchas puertas y ventanas con diferentes opciones para asegurar su futuro. Además, es importante resaltar la aportación de Freire: La educación reproduce su propio plan, la estructura dinámica y el movimiento dialéctico del proceso histórico (Freire, 1967).

\section{Conclusiones}

Los estudios en la antropología resultan totalmente fructíferas cuando se logra conjugar la experiencia, conocimiento e investigación: ejemplo de ello es el trabajo 
de campo realizado en Magueyitos, que permitió apreciar el distanciamiento de la educación y el aprendizaje en la educación rural. Además, hizo posible, al visibilizar las desigualdades sociales, reflexionar y solucionar la dinámica que se tiene desde hace varios siglos reproduciendo en una sociedad donde los informes del Estado mencionan crecimiento educativo desigual, que genera de manera parcializada el acceso a la educación. Es necesario conocer las diferentes realidades sociales existentes para poder realizar investigaciones que ayuden y permitan mejorar la educación, al atender las necesidades de los pobladores y las expectativas que tienen. Para Mendoza, "la alternativa de querer la educación a través de plataformas y programas educativos solo fue resultado de atestiguar grandes brechas de desigualdad social que existen" (2020, p. 2).

Aunque el objetivo del "Programa de Atención Intensiva (PAI)" era muy claro, el tiempo establecido y los recursos eran muy pocos para recuperar lo que se ha venido arrastrando por años, sin embargo, los objetivos establecidos para realizar el trabajo de campo y obtener información para realizar la investigación de manera exitosa dando pauta para más investigaciones por diferentes disciplinas. 


\section{Referencias bibliográficas}

Freire, P. (1970). Pedagogía del oprimido. Río de Janeiro: Paz e Terra.

Galván, E. (2016). Derecho a la Educación. México: Secretaría de Cultura.

Kauffaman, J. (1967). Educación. México: Unión Tipográfica Editorial HispanoAmericana.

Marcelo, C. \& Vaillant, D. (2017). Políticas y programas de inducción en la docencia en Latinoamérica. Cuadernos De Pesquisa, 47(166), 1224-1249. https://doi. org/10.1590/198053144322

Mendoza, L. (2020, junio 19). La Educación en México en tiempos de COVID-19. Educación Futura. https://www.educacionfutura.org/la-educacion-en-mexico-entiempos-de-covid-19/

Navarro, V. (2002). Bienestar insuficiente, democracia incompleta. Barcelona: Anagrama.

Reimers, F. (2000). ¿Pueden aprender los hijos de los pobres en las escuelas de América Latina?. Revista Mexicana de Investigación Educativa, 5(9), 11-69.

Savater, F. (1997). El Valor de Educar. México: Instituto de Estudios Educativos y Sindicales de América.

Schmelkes, S. (Noviembre, 2015). La desigualdad educativa en México. [Diapositiva de PowerPoint]. https://innovec.org.mx/home/images/2-sschmelkes.pdf

Silvia, I. (1999). Elevadas tasas de deserción en latino América.

Tilak, J. (2002). Education and Poverty. Journal of Human Development, 3(2), 191-207.

Venegas, G., Chiluisa, M., Castro, S., \& Casillas, I. (2017). La deserción en la educación. Revista Boletín Redipe, 6(4), 235-239. https://revista.redipe.org/index. $\mathrm{php} / 1 /$ article/view/240 